Nervenarzt $2017 \cdot 88: 711-712$

DOI 10.1007/s00115-017-0353-8

Online publiziert: 8. Juni 2017

๑) Springer Medizin Verlag GmbH 2017

CrossMark

\section{T. Gasser ${ }^{1} \cdot$ W. Maier ${ }^{2}$}

${ }^{\prime}$ Klinik für Neurologie, Abteilung für Neurologie mit Schwerpunkt Neurodegeneration, und Deutsches Zentrum für Neurodegenerative Erkrankungen, Standort Tübingen, Universitätsklinikum Tübingen, Tübingen, Deutschland

${ }^{2}$ Klinik und Poliklinik für Psychiatrie und Psychotherapie, Universitätsklinikum Bonn, Bonn, Deutschland

\title{
Genetische Risikofaktoren für neuropsychiatrische Erkrankungen
}

Die Fortschritte der Genetik haben in vielen Bereichen zu einem grundlegenden Wandel im Verständnis vieler neurologischer und psychiatrischer Erkrankungen geführt. Durch die Entwicklung neuer, sehr viel schnellerer und billigerer Sequenziertechniken hat die Geschwindigkeit dieses Wandels in den letzten Jahren stetig zugenommen. Die Kosten der Sequenzierung des ersten menschlichen Genoms im Humangenomprojekt, das 2001 abgeschlossen wurde, wurden auf über 1 Mrd. Dollar geschätzt. Heute kann ein gesamtes Genom innerhalb weniger Tage für rund 1000 EUR sequenziert werden.

Durch die Erkenntnisse der Genetik wurde klar, dass viele der klassischen Krankheitsentitäten in Neurologie und Psychiatrie in Wahrheit heterogene Syndrome komplexer Ätiologie sind. Die Identifizierung mutierter Gene in monogen erblichen Formen dieser Erkrankung und die Untersuchung der pathologisch veränderten Genprodukte erlauben Einblicke in die molekulare Pathogenese und Pathophysiologie dieser Erkrankungen, was bereits zur Entwicklung erster kausal orientierter Therapiestrategien führt. Damit bieten sich auch für die Neurologie zunehmend Möglichkeiten, Konzepte der „personalisierten Medizin" anzuwenden, die individuelle genetische Prädispositionen mit in Betracht ziehen, um Therapien effektiver und nebenwirkungsärmer anzuwenden.
Psychische Erkrankungen sind (von seltenen monogenen Krankheiten der Kinder- und Jugendpsychiatrie - z. B. Fra-X-Syndrom - abgesehen, die hier nicht behandelt werden) multifaktoriell bedingt mit einer jeweils familiärgenetischen Ursachenkomponente. Dieselbe Ursachenkonstellation gilt auch für häufige neurologische Krankheiten (z. B. Schlaganfall). Moderne Methoden der Molekulargenetik können diese krankheitsspezifischen "genetischen Architekturen" greifbar machen. Genomweite Assoziationsstudien (GWAS) sind dabei die Methode der Wahl; sie zeigen, dass viele der häufigen sporadischen neuropsychiatrischen Erkrankungen genetische Teilursachen in Form häufiger Risikovarianten mit jeweils kleinen relativen Risiken haben. Auch wenn hier die klinische Anwendung in Diagnostik und Therapie noch nicht unmittelbar ersichtlich ist, so zeichnen sich doch Möglichkeiten der Verlaufsprädiktion, der Risikostratifizierung und vielleicht sogar der stratifizierten Therapie auf der Basis genetischer Prädispositionen ab.

\section{》) GWAS machen die krankheitsspezifischen "genetischen Architekturen" greifbar}

Viele Fragen sind weiter ungeklärt. So wissen wir bei vielen der gefundenen ge- netischen Risikofaktoren noch nicht einmal, ob die entdeckte Genvariante selbst, oder aber eine oder mehrere benachbarte Sequenzänderungen für die Risikomodifikation verantwortlich sind und auf welchen molekularen Mechanismen diese Effekte beruhen. Der größte Teil des Genoms, die $98 \%$ der nichtkodierenden DNA-Sequenz zwischen den Genen, ist noch weitgehend Terra incognita. Auch wissen wir noch fast nichts über die Interaktion genetischer Varianten in funktionellen Netzwerken oder über die Zusammenhänge der Genfunktion mit Umwelteinflüssen, wie sie z. B. durch die epigenetische Modifikation der Genexpression vermittelt wird.

Dennoch sind die Fortschritte atemberaubend. Die Fortschritte beruhen vor allem auf zwei methodischen Errungenschaften: a) die zunehmend verbreitete Kultur des „data sharing“ in international kuratierten Datenbanken, die es erlaubt, riesige Mengen genetischer Daten mithilfe neu entwickelter Methoden bioinformatischer Datenverarbeitung sicher und nachprüfbar zu verarbeiten; b) die Erkenntnis der notwendigen Replikation beobachteter Assoziationen zwischen Phänotyp und Genotyp mit dem Ziel des Ausschlusses falsch-positiver Befunde, die angesichts der enormen notwendigen Vielzahl genetischer Marker (ohne konsequente externe Replikation) häufig vorkommen können. Diese Strategien werden in Zukunft weiter verfeinert werden und damit neue diagnostische 
Möglichkeiten eröffnen, die gezielt und sachgerecht eingesetzt werden müssen.

Dieses Schwerpunktheft von Der Nervenarzt soll dazu beitragen, den aktuellen Stand des Wissens um die Rolle genetischer Risikofaktoren für neuropsychiatrische Erkrankungen auf verständliche Weise zu vermitteln.<smiles>CCCCC1CCCCC1</smiles>

Thomas Gasser

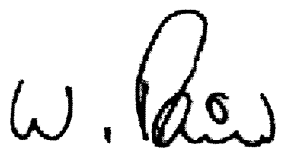

Wolfgang Maier

\section{Korrespondenzadresse}

\section{Prof. Dr. T. Gasser}

Klinik für Neurologie, Abteilung für Neurologie mit Schwerpunkt Neurodegeneration, und Deutsches Zentrum für Neurodegenerative Erkrankungen, Standort Tübingen, Universitätsklinikum Tübingen Hoppe-Seyler-Straße 3, 72076 Tübingen, Deutschland thomas.gasser@uni-tuebingen.de

\section{Prof. Dr. W. Maier}

Klinik und Poliklinik für Psychiatrie und Psychotherapie, Universitätsklinikum Bonn Sigmund-Freud-Straße 25, 53105 Bonn, Deutschland

Wolfgang.Maier@ukbonn.de

Interessenkonflikt. T. Gasser und W. Maier geben an, dass kein Interessenkonflikt besteht.

\section{W. Hewer, C. Thomas, L.M. Drach Delir beim alten Menschen Grundlagen - Diagnostik - Therapie - Prävention}

Stuttgart: Kohlhammer 2016, 309 S., 29 Abb., 27 Tab., (ISBN: 978-3-17-0216174), soft 42,00 EUR

Das vorliegende Werk richtet sich an all diejenigen Leser, die mit der medizinischen Behandlung und Pflege älterer Menschen befaßt sind. Es stellt eine umfassende ÜberDelir beim
alten Menschen
Grundlagen- Diagnostik-Therapie-
Privention
Kohlhanmer sicht zu einem der wichtigsten psychiatrischen Störungsbilder im höheren Lebensalter überhaupt dar. Während für viele Laien ein älterer Mensch plötzlich „verwirrt" erscheint, ist Klini-

kern das Delirium als medizinischer Notfall gut bekannt. Aber selbst Experten werden beim Studium dieses Buches auf eine so sorgfältige Darlegung von Symptomatologie, Ursachen und Therapieaspekten stoßen, daß sie das Buch für ihre Bibliothek anschaffen werden. Dabei muß sich nicht jeder Leser unbedingt mit den spezifischen Problemen der Klassifikation des Delirs auseinandersetzen. Es gehört zum Fach Psychiatrie, daß sich die Terminologie psychischer Störungen immer wieder ändert und die diagnostischen Kriterien im deutschen und angloamerikanischen Sprachraum differieren. Beim Delir wird der Leser erfrischend einfach auf die vier Kernstörungen geführt (Aufmerksamkeit und Ausrichtung der Aufmerksamkeit auf die Umwelt, Wahrnehmung und visuell-räumliche Funktionen, Sprache sowie Gedächtnis und Orientierung), so daß letztlich doch eine hohe Übereinstimmung der unterschiedlichen Diagnosekriterien konstatiert werden kann. Eine besondere Stärke des Buches ist die Darlegung der unterschiedlichen psychopathologischen Erscheinungsbilder deliranter Syndrome im Alter und die sorgfältige Erläuterung spezifischer Untersuchungstechniken, z.B. im Bereich fokussierter und unfokussierter Aufmerksamkeit.

Eine Vielzahl von anschaulichen Kasuistiken unterstreicht die hohe klinische Relevanz dieses Störungsbildes. Dabei wird das Delir-Management bei älteren Menschen im Hinblick auf den demografischen Wandel unserer Gesellschaft an Stellenwert gewinnen: Die Zahl hochaltriger, multimorbider Patienten mit komplexen Erkrankungen und hohem Pflegebedarf wird steigen. Das Problem der sich daraus ergebenen Polypharmazie findet in diesem Werk sehr viel Beachtung und selbst internistisch gut ausgebildete Leser werden beim Studium des Buches neue Einsichten gewinnen und möglicherweise eigene Behandlungskonzepte in Frage stellen. Es stört dabei nicht, dass einzelne Themen (wie z.B. die Exsikkose) in verschiedenen Kapiteln aufgegriffen und bearbeitet werden.

Die besondere Problematik des hypoaktiven Delirs, aber auch andere Aspekte, die zur Nichterkennung von Delirsyndromen führen, werden eingehend erörtert. Die Entwicklung der Versorgungsrealität in den vergangenen Jahren in vielen Kliniken mit immer kürzeren Verweildauern und verstärkter Fokussierung auf eine Hauptdiagnose könnte dazu führen, daß Delirien bei älteren Menschen nicht ausreichend erkannt und behandelt werden. Dem Werk ist daher zu wünschen, dass es nicht nur von Psychiatern gelesen wird, sondern dass es vor allem Verbreitung bei denjenigen Kollegen findet, die primär somatische Erkrankungen älterer Menschen behandeln. Darüber hinaus ist zu wünschen, daß dieses Thema künftig mehr Aufmerksamkeit bei denjenigen findet, die sich mit der Organisation von Krankenhausprozessen und Vergütungssystemen beschäftigen. Delirbehandlung ist möglich, noch besser aber ist Delirprävention. Dafür müssen die entsprechenden Ressourcen finanziert und bereit gestellt werden.

\section{T. Supprian (Düsseldorf)}

